\title{
Understanding and modelling built environments for mobile guide interface design
}

\author{
J. PAAY† and J. KJELDSKOV †* \\ $\dagger$ Department of Information Systems and Faculty of Architecture, Building and Planning, The University \\ of Melbourne, Parkville, Victoria 3010, Australia \\ $\$$ Human-Computer Interaction Group, Department of Computer Science, Aalborg University, DK-9220 \\ Aalborg East, Denmark
}

\begin{abstract}
The research presented in this paper aims to inform interface design for mobile guides by understanding and modelling the built environments in which the guide will be used. This is important because research into the use of mobile guides has shown that people have a strong ability to make sense of the physical space in which they are situated and make use of this when using mobile guides. Based on a field study and architectural analysis of the recently built Federation Square in Melbourne, Australia, we present a descriptive framework, MIRANDA, which provides a summarized abstraction of the fundamental architectural and informational features of a built environment. The use of this descriptive framework in HCI design for mobile guides is exemplified through the design of a mobile guide system for Federation Square that was informed by the identified architectural characteristics. On the basis of the field study and example design, we argue that mobile guides interface design can benefit from making use of 'knowledge-in-theworld' by streamlining and indexing information and functionality to physical information cues implicit in the built environment surrounding the user.
\end{abstract}

\section{Introduction}

Mobile guides are increasingly becoming a part of the way in which we operate in the physical world, and studies suggest that one of the major applications of future mobile information technology will be to digitally enhance user activities by giving access to contextually adopted information through such guides. Hence, the design of mobile guides has received considerable attention over the last decade within the field of human-computer interaction (HCI) (see Abowd et al. 1996, Cheverst et al. 2000, Cheverst et al. 2002, Pospischil et al. 2002). The HCI research literature indicates a broad spectrum of different application areas for mobile guides. Mobile city guides provide the user with maps and other relevant information such as the location of restaurants, public offices, tourist sites etc. adapted to the user's location (Cheverst et al. 2000, Cheverst et al. 2002, Pospischil et al. 2002, Iacucci et al. 2003, Schmidt-Belz and Poslad 2003, Laakso et al. 2003). Mobile guides for specific tourist-sites provide users with the opportunity to follow guided tours or access additional information about the site being visited (Hermann and Heidmann 2002, Bornträger and Cheverst 2003, Bornträger et al. 2003) and mobile museum guides provide additional information about the specific items on display (Aoki and Woodruff 2000, Opperman and Specht 2000, Rocchi et al. 2003). Other mobile guide systems support the user's social or personal life, such as mobile personal guides and event planners keeping track of friends and upcoming social events (Fithian et al. 2003, Kolari and Virtanen 2003, Paulos and Goodman 2004) or mobile shopping assistants keeping track of shopping lists and informing the users about special offers within their vicinity (Randell and Muller 2000, Bohnenberger et al. 2002). Assisting people

*Corresponding author. Email: jesper@cs.aau.dk

Behaviour \& Information Technology

ISSN 0144-929X print/ISSN 1362-3001 online (C) 2005 Taylor \& Francis Ltd

http://www.tandf.co.uk/journals

DOI: $10.1080 / 01449290512331319012$ 
commuting and travelling, mobile navigation guides provide route planning information and directions for wayfinding (Holland and Morse 2001, Chincholle et al. 2002, Kulju and Kaasinen 2002) and mobile travel planners keep track of users' itineraries, upcoming meetings etc. (Ricci et al. 2002, Kjeldskov et al. 2003).

Many of these mobile guide systems involve the user being situated in the built environment of a public space. Yet only a few references have investigated the challenges imposed and opportunities offered by the use of mobile guide systems in the context of buildings and other architectural structures in urban spaces. Exceptions include Vainio et al. (2002) who study navigation and way finding supported by a 3D model-based mobile city guide with clear architectural features such as landmarks, Kulju and Kaasinen (2002) who compare the use of photographs and semi-realistic 3D models augmented with textual information on mobile guides for supporting the user's experience of a physical space, and Laakso et al. (2003) who study the use of realistic 3D maps with topological as well as architectural features as a planning and navigational aid for leisure boat tourists at sea and in the cities they visit. These studies show that people often have a strong ability to make sense of the physical space in which they are situated and typically make extensive use of this when using mobile guides in a built environment. Consequently, it is argued that the use of mobile guides for navigation, way finding, etc. in environments with distinct architectural or other physical features (such as landmarks, noticeable physical structures or special topology) can benefit from taking these features into account by, for example, simply including visual representations of them using street perspective or bird's-eye views (Vainio et al. 2002, Laakso et al. 2003, Nakanishi et al. 2004). However, in order to exploit the user's ability to make sense of architectural features in their physical surroundings in HCI design for mobile guides, we need to better understand the role of the user's physical environment in defining their context and the contribution of existing information embedded into that environment to people's experience of it (Agre 2001, Bradley and Dunlop 2002, Tamminen et al. 2003). Also, we need to learn how to make a clear connection between the user's physical surroundings and the information presented on their mobile guide (Dix et al. 2000, Persson et al. 2002). So far, systematic methods for gathering, analyzing and understanding the properties of a built environment that help define the user's physical context, and appropriate analytical abstractions suitable for informing interaction design on the basis of this, have not yet been developed.

\subsection{Indexicality: relating interfaces to their context}

An interesting approach to making a clearer relation between mobile device interfaces and the user's context is to apply the idea of indexicality. Indexicality is a concept drawn from semiotics describing the relation between information representations and the context in which an interpreter (user) perceives them. Semiotics operates with three types of representations: symbolic (conventional), iconic (similarity) and indexical (material/causal). Symbols and icons are ways of representing information independent of context like, for example, text and graphical illustrations. Indexes, on the other hand, are ways of representing information with a strong relation to, for example, their spatial and/or temporal context exploiting information present in the interpreter's surroundings. Thus, indexical representations are highly context-specific and only make sense in particular situations; at a specific time, in a specific location, in relation to a specific activity, to specific people, etc. As an example, signposts and information boards are typically highly indexical in the sense that their meaning is tied to a specific location. By locating information in time and space, symbolic and iconic representations can be converted into temporal and spatial indexical representations (Andersen 2001). As shown by Kjeldskov (2002), increasing the level of indexicality typically results in a significant reduction of required symbolic and iconic representations.

The concept of indexicality has previously been applied to the design of mobile guide interfaces (Kjeldskov 2002, Graham and Kjeldskov 2003, Kjeldskov et al. 2003) in order to streamline the information and functionality delivered to the user. The idea of applying indexicality to interface design for mobile guides is that if information and functionality on a mobile guide can be indexed to the user's context, then information already provided by the context becomes implicit and does not need to be displayed by the system. Hence, the user's environment becomes a vital part of the interface. This way of making use of 'knowledge-inthe-world' (Norman 1990) allows the limited screen real estate of mobile guides to be optimized to contain only the most vital content and the required user interaction with the mobile guide to be reduced. As a simplified example of this, an indexical mobile guide for patrons entering a cinema complex could be made temporally and spatially indexical by taking into account the time and location of the user, providing only information about the upcoming movies playing within a limited frame of time (temporal indexicality) in that specific cinema (spatial indexicality) (Kjeldskov 2002).

In the studies mentioned above, the mobile guide interface designs only explored temporal and spatial indexicality. However, the idea of indexical interfaces could also be broadened to exploit other aspects of the user's context. In addition to time and location, for example, factors such as existing information in our surroundings, the features of the built environment itself and the use of that environment by other people also play an important 
role in the context surrounding a mobile user (Paay 2003), and may also be indexed to in a mobile guide.

Relying heavily on the user's knowledge about, for instance, when, where and why they are situated, successful design of indexical interfaces for mobile guides require that HCI designers have a strong understanding of the aspects of the user's context that they are indexing to. Hence, in order to include indexes to the built environment in the design of a mobile guide, the features of the built environment contributing to the user-experience of the physical space in which it will be used, needs to be analyzed and modelled in a way that extracts the essence of the place and provides an overview. Information embedded into the built environment and carrying parts of its meaning through its architectural features needs to be identified and proposals for how this can be applied to mobile guide design needs to be developed and evaluated.

This paper contributes to this discussion by presenting 1) a possible approach to enquiring into the features of a built environment informed by theories and methods derived from architecture, 2) a method for creating a descriptive abstraction on the basis of the field data, and 3) an example of how this abstraction can be used to inform the design of a mobile guide prototype. In section 2 , we present and discuss the architectural theories and methods used in the study. In section 3, we report from a field study into the architectural and informational properties of a specific built environment, Federation Square in Melbourne, Australia. The idea and early results from this study are described by Paay (2003). The outcome of the field study is presented in the form of a descriptive framework for modelling and understanding the built environment called MIRANDA, which captures positive and negative architectural and informational properties of a physical space. In section 4, the use of this descriptive framework in HCI design for mobile guides is exemplified through the design of a mobile guide system for Federation Square, in which the interface is indexed to the built environment by exploiting information already implicitly present in the user's physical surroundings. Finally, section 5 concludes our work and outlines our present and future directions of research.

\section{Analyzing the built environment}

Architectural design has a history of incorporation of social theories and user needs into design methods. Of special interest, urban planner, Kevin Lynch (1960) and architect, Christopher Alexander (Alexander et al. 1977) both modelled built environments, specifically cities, with regard to the people that inhabit those places, hence implicitly including the users in their analysis of physical space.

Lynch (1960) developed a method for visual analysis of city precincts through descriptions of key aspects of the space held by people as they navigate and orient themselves within city precincts. This was done by diagramming the interplay of visible elements in the environment that contribute to a person's environmental image of a place. His purpose was to develop ideas and methods, rather than to prove facts in a final and determinate way. The method proved successful at assisting in the analysis of types of elements of a city, how they are put together, and what makes for strong identity. It also proved to be a useful technique for predicting the probable public image of that city. Lynch documented this technique for describing and understanding key aspects of the environmental image of space held by people as they navigate and orient themselves within city precincts and noted that it would be interesting to apply this method to an environment of a different scale. To understand the role of environmental images of cities in the lives of those who inhabit them, Lynch carried out two basic analyses. Firstly, interviews were conducted with people who either lived or worked in that area. The city dwellers image of the city was elicited from them using interview techniques which included asking participants to describe features of the city from memory, asking them to draw sketches of the city, and having them make imaginary trips to destinations within the city precinct. Secondly, a field reconnaissance was done by an architecturally trained observer, who mapped the presence of various elements of the physical environment, using categories that had proven significant in analysis of earlier pilot interviews: districts, landmarks, nodes, edges and paths. The observer made subjective judgments based on the immediate appearance of these elements in the field and about their visible contribution to the image of the city, which resulted in maps representing the visual form of a city in respect to these five elements. These maps were then compared to those maps derived from consensus information collected in the verbal interviews and the sketch maps made by the inhabitants to draw conclusions about 'imageability' of built environments.

Alexander et al. (1977) empirically investigated the interplay between architectural space and its inhabitants and identified architectural design problems in context and their impact on inhabitants of that environment. Drawn from observations of historical solutions to common design problems, he created a method of analyzing aspects of the built environment and generated a 'checklist' of plausible solutions for design. This checklist was constituted by a collection of 253 hierarchically ordered patterns making up a 'Pattern Language' (Alexander et al. 1977) that begins with patterns defining towns and communities, through the design of individual buildings, down to the detailed design of building elements. The pattern language provides a framework for solutions to these common design problems. Each pattern describes the field of physical and social relationships required to solve a design problem in its stated context. These patterns in themselves are a form of 
composite pictures including: photographs, sketches, descriptive explanations detailing the context for the pattern, its relationship to parent patterns, a description of the problem, the empirical background of the pattern, evidence for its validity, and the design solution.

The methods of Lynch (1960) and Alexander et al. (1977) have not only proven their value within architecture, but have also demonstrated usefulness in $\mathrm{HCI}$ research in analyzing the interplay between architectural, informational and social space with the purpose of designing computerized information systems.

The work of Lynch (1960) is often referenced in relation to the design of computer systems supporting orientation and navigation in complex virtual information spaces, virtual environments and in the real world, and is claimed to be one of the most influential and useful pieces of research on spatial orientation and navigation for interaction designers (Sparacino et al. 2000). Information City (Dieberger and Frank 1998) is an ontology of spaces and connections based on Lynch's five major elements developed to support navigation in large information spaces where a spatial metaphor supports a user's sense of orientation by the use of recognizable landmarks. Similarly, Sparacino et al. (2000) present a 3D web browser using an urban-like information landscape following the guidelines proposed by Lynch (1960) to fetch and represent information from the web. Supporting navigation in virtual reality, Ingram and Benford (1996) apply urban planning principles to the design of virtual environments using Lynch's work to improve the navigation of a virtual environment including districts, landmarks, paths, nodes and edges. Vinson (1999) proposes a series of guidelines for how designers can create and place landmarks in virtual environments for supporting navigation and orientation. Informing the design of a mobile guide system, Kulju and Kaasinen (2002) draw on the work of Lynch (1960) to include landmarks in a 3D city model on a mobile device and augment them with additional textual information to optimize spatial orientation.

The work of Alexander (Alexander et al. 1977) has been successfully applied within various areas of computing; from object-oriented software design (Gamma 1996) to human-computer interaction (Tidwell 1999, Borchers 2001). Inspired by, among others, Alexander et al. (1977), Harrison and Dourish (1996) define a distinction between space and place, and illustrate the influence of peoples sense of place on their interactive behavior. Designing information technologies for the domestic environment, Crabtree and Hemmings (2001) use an adapted pattern framework to inform requirements analysis and describe day-to-day use of technologies within that environment. Informing novel design of pervasive and mobile device services, Paulos and Goodman (2004) enquire into the application of new technologies by city inhabitants, and draw an analogy between Alexander's patterns and the movement and activities of people in urban settings. In line with this research, McCullough (2001) points out that as interest in information technologies for physical contexts increases, what we already know from the work of people such as Alexander about the built environment and how people operate in it, can inform our understanding of peoples' situated interactions, and guide the design of technologies that consider the user's physical context.

\section{Field study: Federation Square}

Inspired by Lynch (1960) and Alexander et al. (1977), we conducted a field study of the architecturally designed built environment at Federation Square, Melbourne, Australia.

Federation Square (figure 1) is a new civic structure, opened to the public in October 2002, to provide the people of Melbourne with a 'unifying square, a landmark, a civic focus' (official brochure), by bringing together a creative mix of attractions and public spaces. The design intention for the space was to incorporate digital technologies into the building fabric creating a meeting of virtual information space and physical building space for people to experience. We chose Federation Square for this study because it is a multi-modal public space with a mixture of distinct architectural features and embedded digital elements that provide a variety of activities to visitors.

The aim of the field study was to inquire into how informational and architectural elements of the built environment contribute to the visitor's experience of a public place, and how this could subsequently be modelled. Thus, we wanted to identify important properties of the built environment as an inhabited public space and create an analytical abstraction, which could inform the design of a mobile guide supporting visitors to this place. The result of the field study is a descriptive framework called MIRANDA (Multilayer Information Related to Architecture aNalysis Data Abstraction), representing the human experience of the informational (analogue and digital signage) and architectural properties of a physical space. The result of the subsequent prototype design is a proof of concept for a mobile guide which takes these properties into consideration.

The study consisted of the following sequential activities:

- Field inspection of Federation Square.

- Data coding (based on Lynch (1960) and Alexander et al. (1977)).

- Data analysis.

- Data synthesizing: developing MIRANDA.

- Development of mobile guide prototype design.

The field inspection took approximately three hours, data coding took eight hours, and data analysis took approxi- 


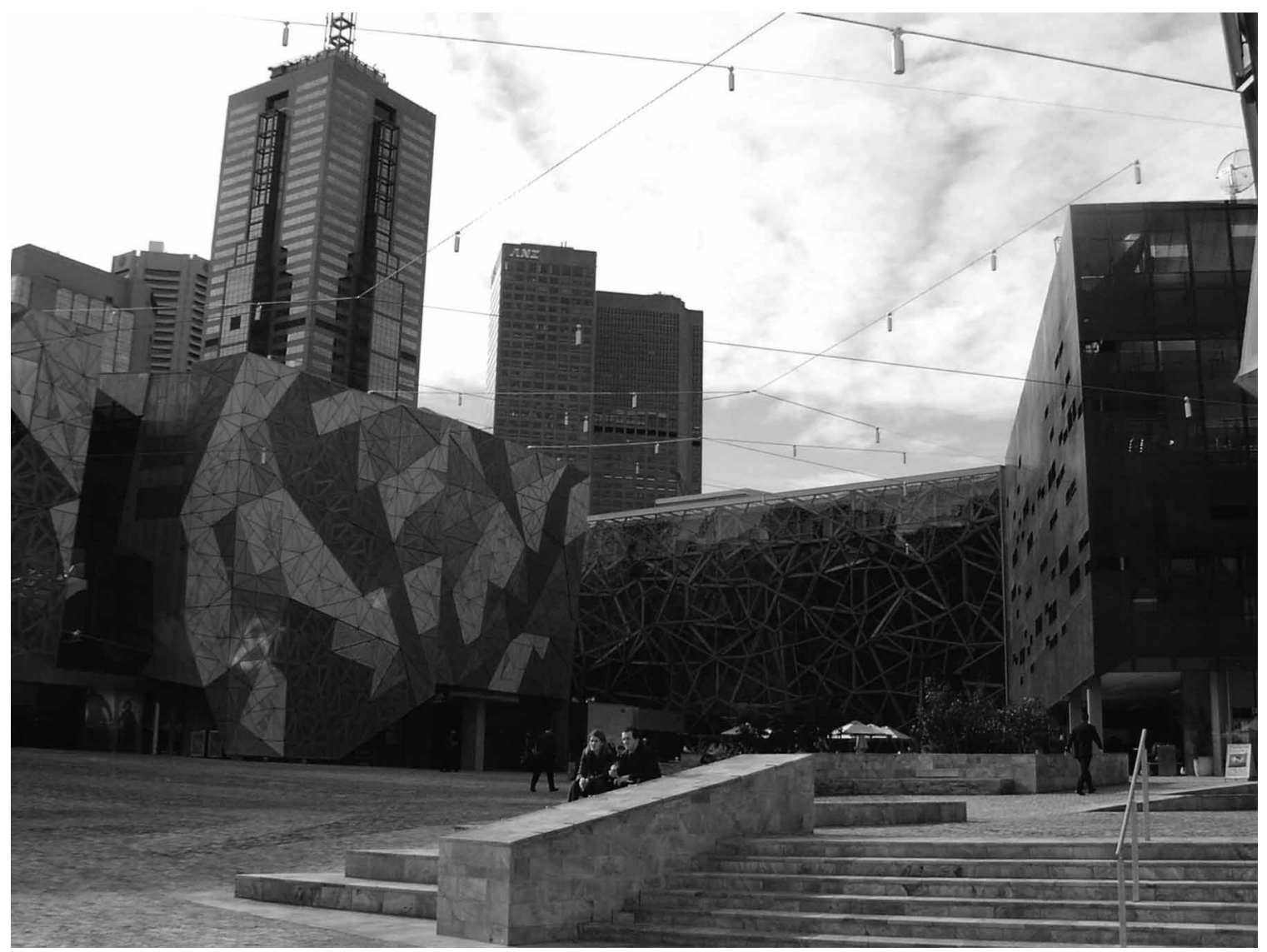

Figure 1. Federation Square.

mately three days. Synthesizing the data took eight hours and developing the preliminary prototype design took approximately two days. Although these estimates can serve as guidelines for similar analysis by other mobile guide designers, the exact time spent on these activities would depend on the size of the physical site being analyzed.

The details of these activities are described below.

\subsection{Inspecting Federation Square}

An initial visit was made to Federation Square by the first author, and resulted in the development of a scenario of the experience of a first-time visitor to the square. Observational expert audits were then made of Federation Square for both architectural and information space, in two separate field visits. These expert audits were inspired by the method outlined by Lynch (1960) in his visual analysis of city precincts for the purpose of identifying types of elements of a city. In his method, an architecturally trained observer maps the presence of various elements of the physical environment. In this adapted method, the trained observer records, through photographs and field notes, the elements of the physical environment for later classification using significant categories. These visits, each taking about three hours to complete, resulted in a collection of 250 digital photographs of physical elements of the built environment. The location of each photograph was recorded on a map of Federation Square, and corresponding observations of the relationship between the elements being photographed and the environment (including interaction with people inhabiting the space) were recorded in the form of field notes.

\subsection{Coding the data}

The photographs were stored electronically in a format where they could be directly associated with their corresponding observational field notes and sketches, and could be annotated further during the process of coding and analysis. For the information elements, each sign and media screen was sketched showing its relationship to building fabric, viewing direction and distance, and then annotated with general field notes explaining the sketches and detailing auditor observations of human activity in relationship to the signage. For the architectural elements, field notes recorded observations made by the trained 
auditor including general descriptions of elements and human responses to physical spaces within the architecturally designed environment. The coding of this data was necessarily a two-phase process, given that the original source data was a mixture of images and descriptive text. The first pass through the data used the encoding schemas to convert classified elements of the images into corresponding text. The second pass combined the classificationgenerated image descriptions with existing observational field data and highlighted those aspects of the resulting prose that contributed to the encoded abstraction of that data.

In coding the information elements, the graphic communication theories and concepts of Bowman (1968), Tufte (1990) and Bertin (2003) were used to derive the following set of classifications for the information elements: type, direction, distance, visibility, readability, and location. These categories were used as an encoding schema, to group and order descriptions of sketches and field notes in the electronic file. The ordered descriptions were then read through in their entirety several times, and repeating phrases and key concepts associated with the encoding schema were highlighted in the descriptions associated with the images. Bowman (1968), Bertin (1983) and Tufte (1990) were used to develop the categories for the analysis of information space because their graphic communication theories are based on human interpretation and human understanding of graphic elements in signage, and therefore necessarily incorporate human experience of information into the analysis.

The architectural elements were classified initially using Lynch's (1960) categories of: district, landmark, node, path, and edge, as an encoding schema. Using Lynch's detailed descriptions of what constitutes each of these categories, the key element of each image was classified in respect to the schema, by the architecturally trained auditor using a visual inspection technique. The photographs were annotated with a legend indicating their association with one or many of the five categories. An additional classification of this data was then conducted on the architectural elements using the 253 patterns of Alexander's pattern language as an encoding schema. Sketches and notes showing the applicability of each pattern were appended to the existing field notes. Each image was associated with one or many Alexandrian patterns, and the photograph annotated with the pattern number and pattern title of each associated pattern. The text descriptions associated with each architectural photograph were then read through several times to identify key concepts and repeating phrases in the loosely structured data that related to the encoding schema. These key themes were highlighted in the text data.

Lynch (1960) and Alexander et al. (1977) were used to derive the encoding schemas for this analysis because the categories that they provide were the outcomes of empirical studies of the interplay between architectural space and people, and this study aims to understand and model the human experience of a place.

\subsection{Analyzing the data}

Using a form of content analysis adopted from rapid ethnographic method (Millen 2000), the highlighted concepts and themes were extracted from the descriptive prose, and analysed to see if some kind of 'vocabulary' of the space would emerge.

The architectural data was analysed first. The Lynchian categories derived from the image data were directly mapped and overlaid onto an existing two-dimensional map of Federation Square to produce a colour-coded abstraction indicating the location of the environmental categories. From this diagram it was clear that four key districts and four key landmarks could be identified in the complex. District 1 is a transit zone, a connector to the city, and has a focus on Landmark 1, the information centre. District 2 is the main plaza, an earthy uneven textured sloping open space with outdoors activities, which focuses on Landmark 2, the stage and large media screen. District 3 is the atrium precinct, sheltered, noisy, and constructed of machine-made materials. Its focus is Landmark 3, the entrance, a large opening to this area. District 4 is the river precinct, which has the feeling of being at the back; flat, damp and lower than the rest of the spaces, Landmark 4, the river, is the focus of this district (see figure 2).

The next stage was to use affinity diagramming adapted from the contextual design methodology (Beyer and Holtzblatt 1998) to group and refine the repeating phrases and key concepts that were identified in the data through the encoding process. Each phrase and word was written on a post-it note, and these notes were stuck on a large white board. After several iterations of grouping, regrouping, forming sets of words and refining words to a concise set of representative terms, the following themes emerged: words that described the space in terms of how it felt to people (affect), words that gave rich descriptions of architectural features, words that described the location of elements in relation to each other, and words that described human activity in the space. The emerging words were, by virtue of the encoding schema, influenced by the categories of Lynch (1960) and Alexander et al. (1977).

The same process of affinity diagramming was used with the informational data, and the following themes emerged: words that related to the readability of signs, words that related to the visibility of signs, words that related to the physical location of the sign, words that described how people were using the signs, and words that described the quality and usefulness of information given (including the understandability and effectiveness of the information). 


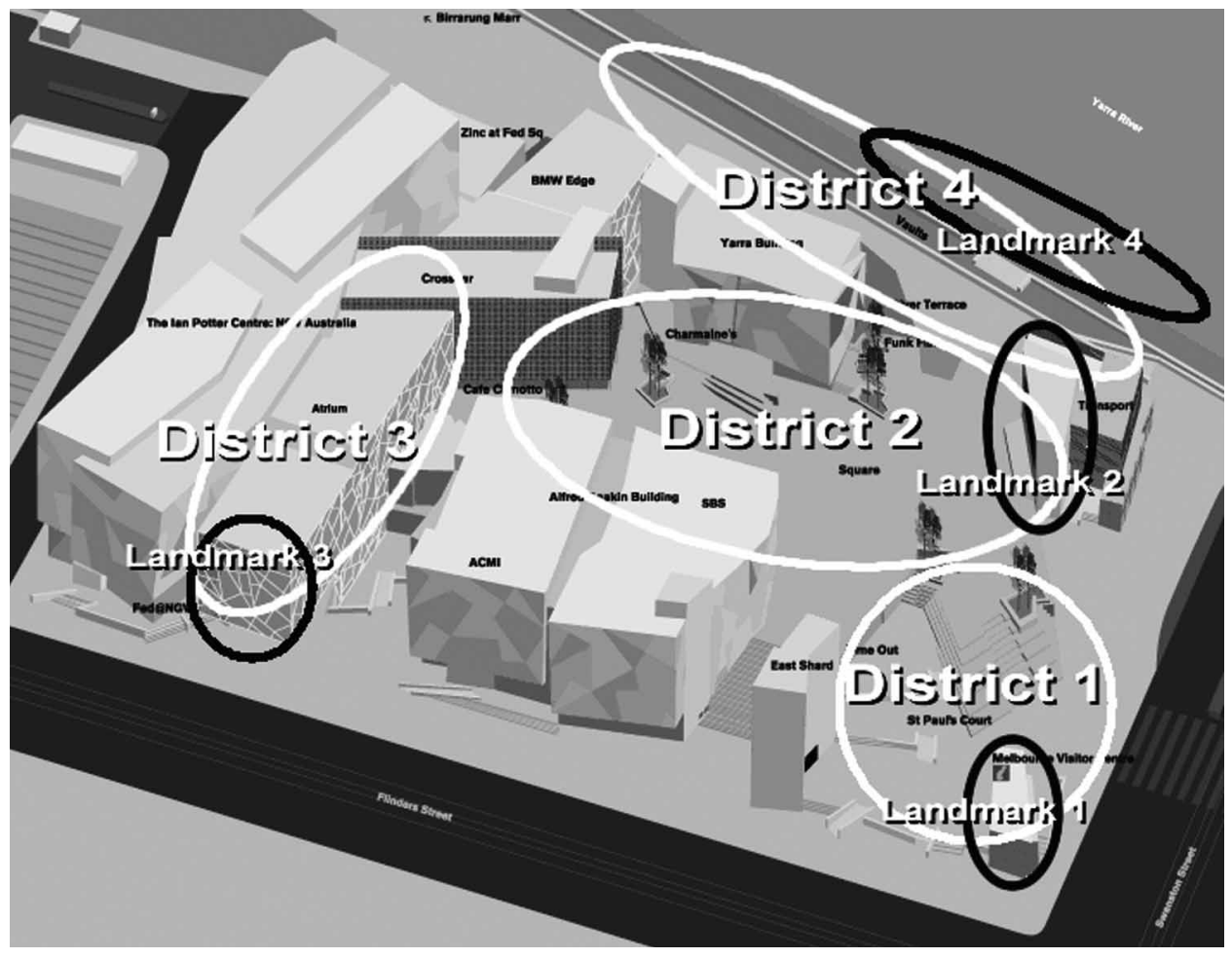

Figure 2. Federation Square: district and landmarks.

The emerging words were, by virtue of the encoding schema, influenced by the classifications and descriptions of graphic communication used by Bowman (1968), Bertin (1983) and Tufte (1990).

\subsection{Synthesizing the data: MIRANDA}

The process of grouping, sorting, dissecting and refining word sets using the affinity diagramming method had resulted in an emergent 'vocabulary', for both the architectural and the informational data, which described the physical environment using a concise set of words and clarified and identified the essence of the characteristics of the space. The process had been iterated until the syntax of a 'language' and a stable and concise set of words, representing the semantics of that language, evolved. This language was necessarily refined to a point where it could be used to completely replace the text descriptions associated with each of the images, and hence be used to represent a concise and abstracted description of that space, providing a minimalistic representation of human understanding of that space.

The syntax of the language, MIRANDA, became a signed word-pair:

$[+, /,-]<$ descriptor $>.<$ place $>$

Explaining this syntax, the first set indicates that only one of these signs, ' +', ' $/$, or '-', applies to the phrase, where ' + ' indicates the positive form of the phrase, '" indicates the in-part form of the phrase and ' - ' indicates the negative form of the phrase. The sign is then followed by a word-pair, the 'descriptor' and 'place' words, each chosen from a finite set of describing words, and a finite set of place words, which were defined during the context analysis phase of the space. Together, they give the adjective and noun for a 'language' statement that can represent the human understanding of the environmental element being described. For example, '+ inviting.path' indicates that an inviting path is pictured, whereas 'inviting.path' indicates that the path is uninviting to people. The two sets of words that represent the architectural characteristics of Federation Square, in respect to the expert audit can be seen in figure 3 and 4 , where descriptor words are on the left-hand side, and place words on the right-hand side.

The same synthesis process was completed for the information elements. The syntax of the information language that was emerged was:

$[+, /,-]<$ descriptor $>.<$ attribute $>$

To confirm the richness of MIRANDA, one or many language statements were then used to replace the detailed prose description associated with each photograph to ensure that the key element of every image could be comprehensively described using the language. For example, the description associated with figure 1 was replaced by 


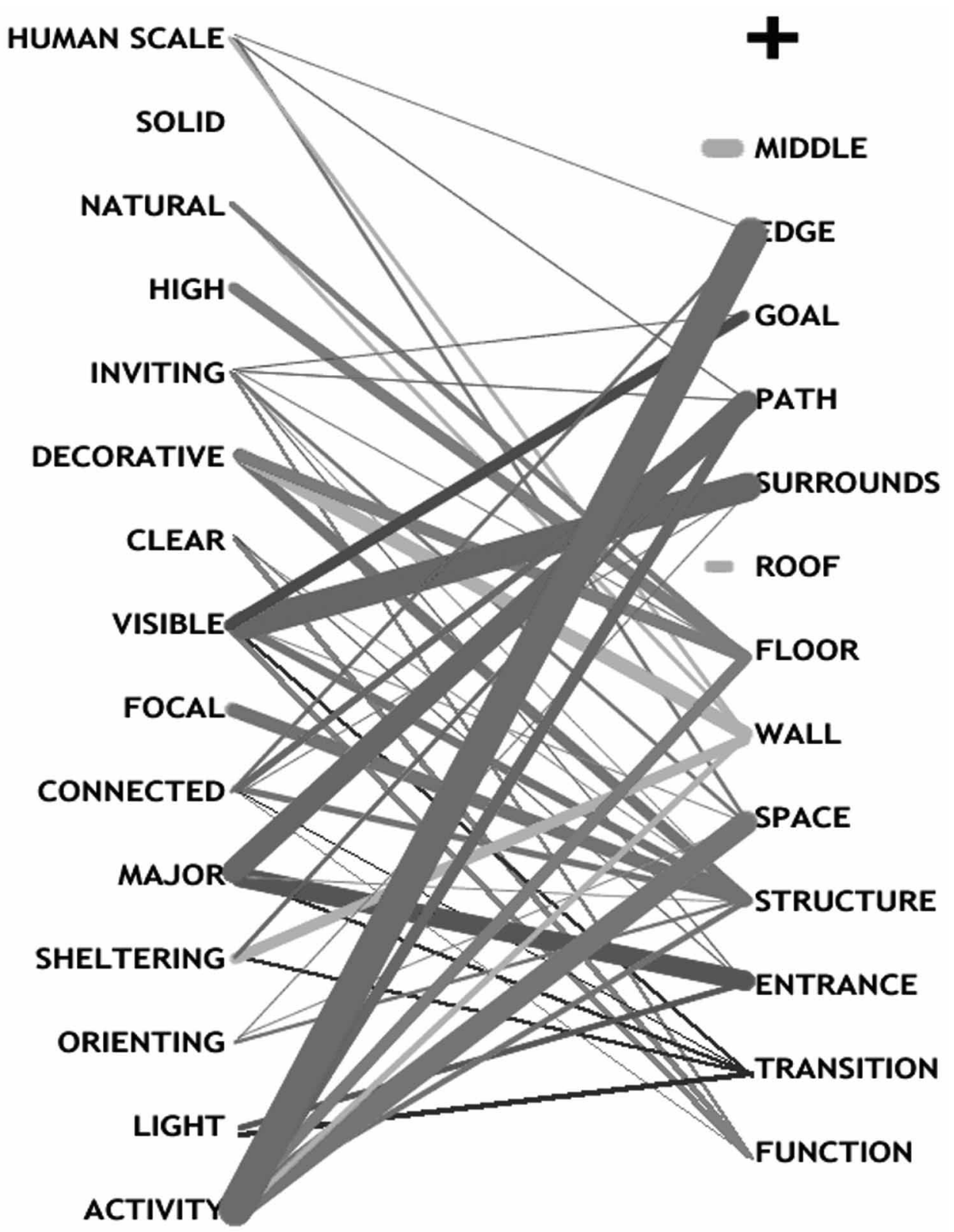

Figure 3. Abstraction of MIRANDA: positive description of architectural characteristics.

the language statements: '- clear.path', ' + activity.floor', and '- activity.middle'. These collected statements can be interpreted to indicate that the paths in this area are undefined, the paving on the ground and the steps in this area are primarily used as places to sit and watch, and the middle of the space is clear of activity. In the interest of a concise descriptor set, the word 'activity' was a composite descriptor that emerged from the grouping process, representing the following different types of activities that were extracted from the image descriptions: walking, sitting, strolling, meeting, watching, being seen, shopping, and dining. Future iterations of the language may have to include these more detailed activity descriptions, depending on the granularity of its use. To confirm the succinctness of MIRANDA, word pairs were associated diagrammatically using the abstraction shown in figure 3 and 4 to visualize variations and frequencies of word pairs.

This was done using layered drawing software, with each different coloured layer representing the links from a signed place word, to its associated set of descriptors. This made it possible to view different combinations of word pairs for comparisons with each other, and to make it possible to view all pairs simultaneously. Each occurrence of a word pair within the dataset was plotted on the diagram as an 


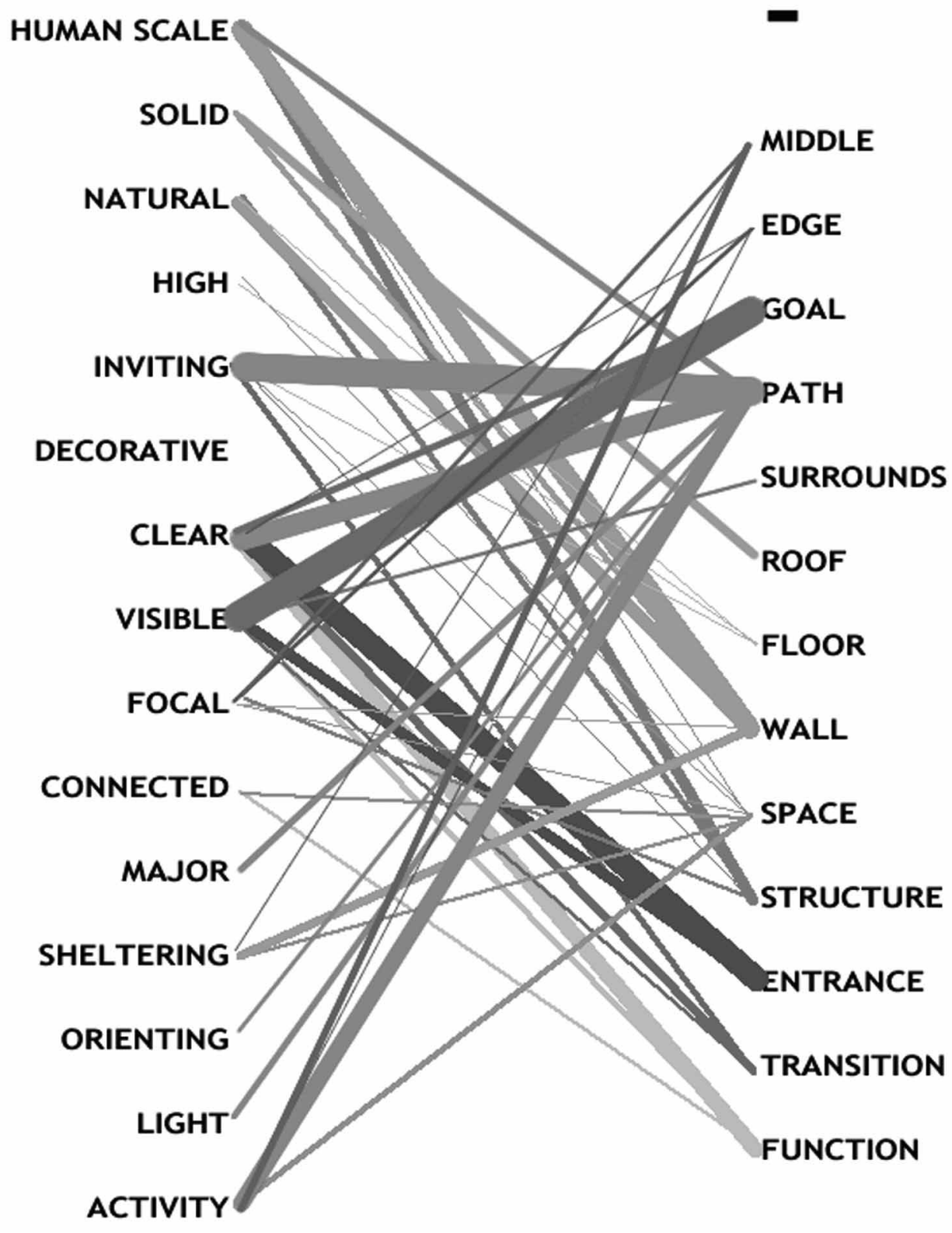

Figure 4. Abstraction of MIRANDA: negative description of architectural characteristics.

additional one-point line width to the connecting link between the words. When all the language statements had been plotted on the diagram, the width of the word pair link therefore represented the number of occurrences of this word pair in the total dataset. This gave a visual form to the 'language' and helped to validate the choice of words in the finite word sets by indicating whether word-pairs were actually used in describing the space, the existence of a link, and frequency of use of those pairs, and the width of the link.

This abstraction of architectural characteristics provided an additional benefit as a tool for visualising a summary of human experience of architectural elements of that environment, that is, an overview of the physical context of that space. In surveying the diagram, it is possible to draw summary conclusions about the space which would not be evident from viewing the original data, or from merely visiting the space, because it represents a composite view of that space, judiciously extracted from historical understanding of human experience of architectural space, as a lens for expert observation and analysis of this built environment.

MIRANDA was also applied to the information layer of Federation Square (analogue and digital signage), but for 
the purpose of clarity and exemplification of indexing to physical context in mobile guide interface design, the remainder of the paper will focus on the outcomes of the architectural analysis as illustrated in figure 3 and 4 .

In analyzing figure 3 , it is evident that activity in Federation Square occurs primarily along the edges and spaces generally have little activity in the middle. This can be surmised because the link between 'activity' and 'edge' is thick ( + activity.edge). We can also infer that the square looks out at the views of its surroundings, evidenced by the thick link between 'visible' and 'surrounds' ( + visible.surrounds). Many of the spaces have activities associated with them, such as places that are designed specifically for an activity such as sitting or dining, and so the link between 'activity' and 'space' is moderately thick ( + activity.space). Similarly, landmarks can be used to orient oneself, from most places around the square, indicated by the thick link between 'focal' and 'structure' ( + focal.structure). Other moderately thick links can be seen between 'major' and 'entrance' (+ major.entrance) and 'major' and 'path' ( + major.path), indicating that Federation Square has a few main entrances into buildings and main pathways around these structures, as opposed to a network of small pathways and multiple openings you might find in the centre of an old city.

Additionally, figure 4 enhances our understanding of the space by representing additional summary information about it in the form of the negation of these word-pairs. For instance, those major paths, that is, pedestrian links between two key destinations in Federation Square, are not inviting ( - inviting.path). They are often either a narrow gap between two tall walls of adjacent buildings ( - humanscale. wall) or they are down badly lit dark underground corridors ( - light.path). When you are trying to find a specific place within the complex it is often difficult to see your destination, in several places you begin to follow a sign to a specific place, but the place that you are aiming for is not visible through the entrance indicated, leaving visitors unsure that it is the way to go ( - visible.goal). In many places the pathway that you are supposed to take is unclear ( - clear.path) because paths are not specifically indicated in open spaces.

This visual abstraction of MIRANDA represents a descriptive framework for understanding and modelling the physical context of a public place. MIRANDA makes available a vocabulary of a specific space that can then be used to understand the key physical characteristics of that built environment, and to describe the user's surroundings in a way that is grounded in human observation of that place, and formed with reference to collected knowledge about human understanding of architectural form and graphical communication. MIRANDA is unique, in so far as it is a systematic tool for creating an abstract representation of physical context, which does not exist in the literature surveyed, and yet that same literature often laments a lack of in-depth understanding of the physical context for which pervasive information systems are being designed. The aim of creating MIRANDA is to provide mobile system designers with knowledge about elements in the user's physical context, so that information that already exists in the world can be indexed to in the interface. The use of MIRANDA for this purpose is exemplified below.

\section{Mobile guide design}

To explore the contribution of MIRANDA to the design of physically indexed interfaces, we have designed a mobile guide system for use at Federation Square as a proof of concept. The design presented below is currently being implemented as a functional prototype running on handheld computers using Bluetooth for positioning within districts and GPRS for wireless access to the internet. The prototype will be lab and field-tested early 2005 .

The design of the prototype guide system is based on a combination of the findings from the Lynchian analysis of Federation Square and MIRANDA. The Lynchian analysis showed that clear districts can be identified within the space of Federation Square. MIRANDA showed for each of these districts, key architectural characteristics can be identified on a level of abstraction that allows a conceptual image of the space rather than a catalogue of specific physical elements.

Combining the findings from the Lynchian analysis and MIRANDA, we have developed three overall design ideas for a mobile guide for Federation Square, which exploits unique characteristics of the physical space and indexes to features of the built environment:

- The mobile guide responds to the user's location in terms of one of the defined districts rather than Cartesian coordinates.

- Each district is represented in the mobile guide by an interactive photorealistic depiction of the physical surroundings augmented with textual or symbolic information needed to better understand the place.

- Locations and instructions for navigation are expressed through rich descriptions derived from the distinctive characteristics of the place rather than through Euclidian coordinates.

The implementation of three design ideas in our mobile guide system is detailed below.

\subsection{Location by district}

In our mobile guide for Federation Square, the user's location is defined by four districts. These four districts were identified by the Lynchian analysis, each with their own distinct characteristics and a corresponding landmark. The location districts we are using make use of people's ability to make sense of the physical environment in which 
they are situated and are not defined by coordinates but by the human experience of the physical layout of the space. Basing positioning on location within a limited number of districts rather than on exact $\mathrm{x}, \mathrm{y}$ coordinates also makes it possible to implement positioning using, for example, Bluetooth beacons rather than GPS.

The location districts of the mobile guide for Federation Square and corresponding screens are illustrated in figure 5.

The information pushed to the mobile guide is tailored to information needs within a specific district. When a user moves into another district, their context changes, and so does the information that appears on the screen of their mobile device. To allow the user to align the information in the guide to their physical surroundings, the initial screen displays the corresponding landmark for that district.

In the following subsections, we describe the specific design of the screens associated with only one district: the main plaza.

\subsection{Augmented interactive photorealistic depictions}

When the user enters a district, an interactive photorealistic depiction augmented with textual and symbolic information pertinent to that district is pushed to their device. For the main plaza, MIRANDA identified six central architectural characteristics:

- Activity edges.

- Focal structures.

- No visible goals.

- Visible surrounds.
- Major entrances and paths.

- No clear paths.

These six features informed the interface design of the screens for the main plaza.

Wishing to represent the main plaza district in the system, we chose to use a $360^{\circ}$ panoramic depiction of the plaza because MIRANDA tells us that the main plaza has 'activity edges' and is open in the middle. Focusing on the edges of the plaza will match the guide to the user's experience of the physical space and allow them to easily align the information presented in the system with their physical surroundings. We use photorealistic depictions in the design because we also know from MIRANDA that the main plaza has 'focal structures'. This enables users to align the image in the interface to the architectural features of the built environment in their current district (for example, as seen in Pospischil et al. 2002). Subsequently, they can easily scan through the virtual representation of the space and index the information presented in the mobile guide to the corresponding physical objects in their surroundings. Like when operating a QuickTime VR movie, panning is done by pointing on the panoramic depiction and dragging in the desired direction.

In a situation where the physical analysis of a district indicated that features of interest were, for example, clustered or spread out over a very large physical area, it might be more appropriate to use a different depiction such as a bird's eye view approaching the user's perception of that space (Kulju and Kaasinen 2002, Laakso et al 2003).

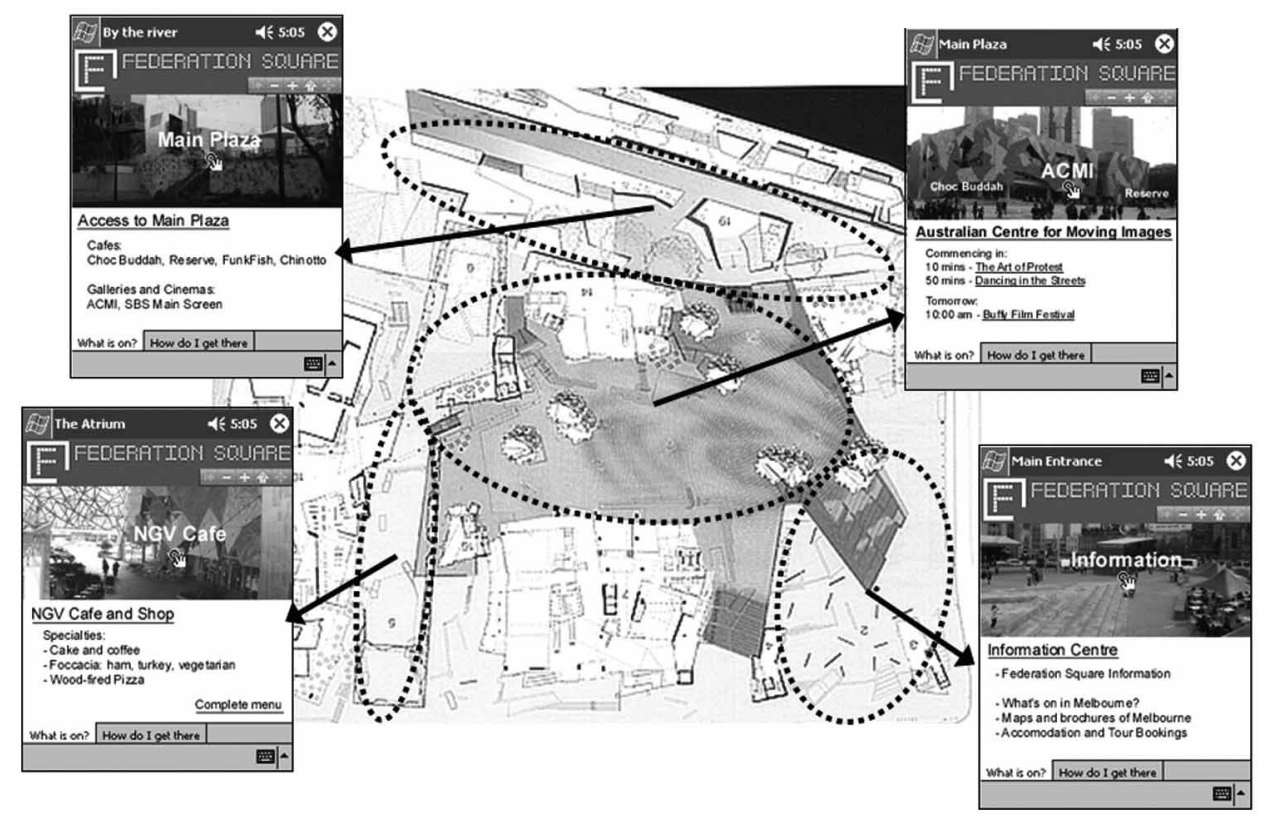

Figure 5. Location districts of the mobile guide for Federation Square and corresponding screens. 


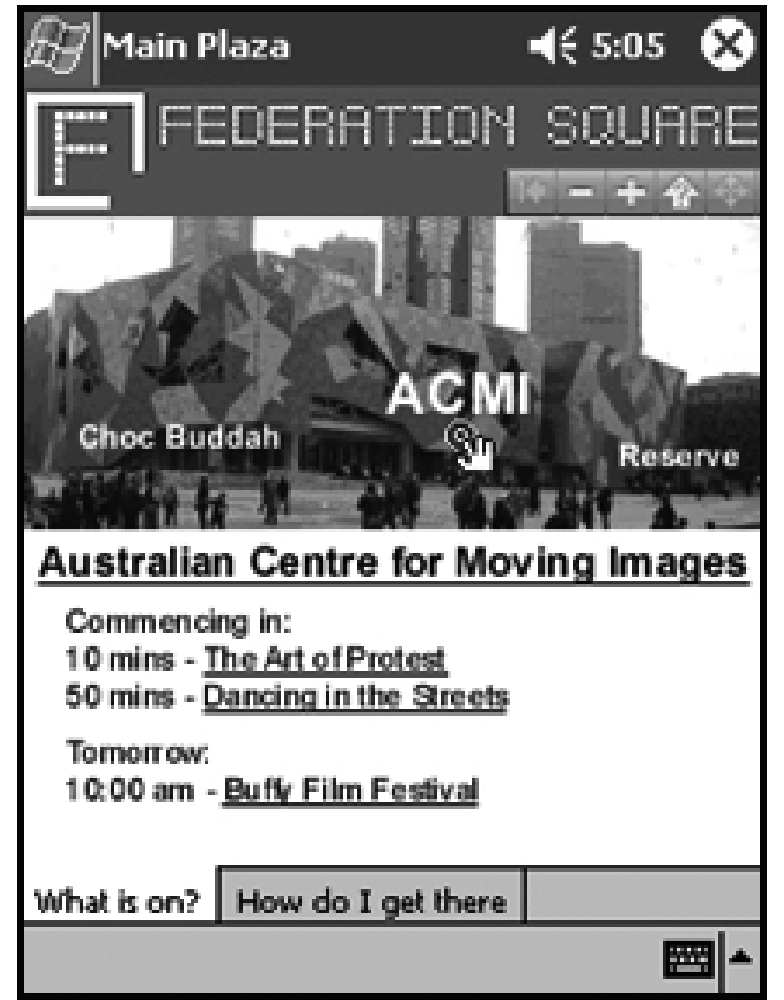

Figure 6. Mobile guide for Federation Square: location and event finding screen.

The main screen for the main plaza is shown in figure 6. MIRANDA also identified a characteristic of the space as having 'no visible goals'. This feature of the space led to the augmentation of the $360^{\circ}$ panoramic depiction of the plaza with white text labelling visitor destinations not clearly indicated by the architectural design. In many cases, the actual location of activities lies behind the visible facades, but the facades function as clear 'focal structures' that can be indexed to in the system, linking information provided by the system to those visitor destinations. A location is automatically selected when it is positioned in the centre of the display. This is indicated by the enlargement of the text label of that location and by displaying the associated detailed information, as shown in figure 6. From this screen, the user can access additional details by clicking on the underlined links. Access to navigation information about that location is also now available by clicking on the 'How do I get there?' tab.

\subsection{Rich descriptions for navigation}

When the user clicks on the 'How do I get there?' tab from the main screen, they are presented with location and navigation information about the selected destination. The design of the navigation screen, as shown in figure 7 , was

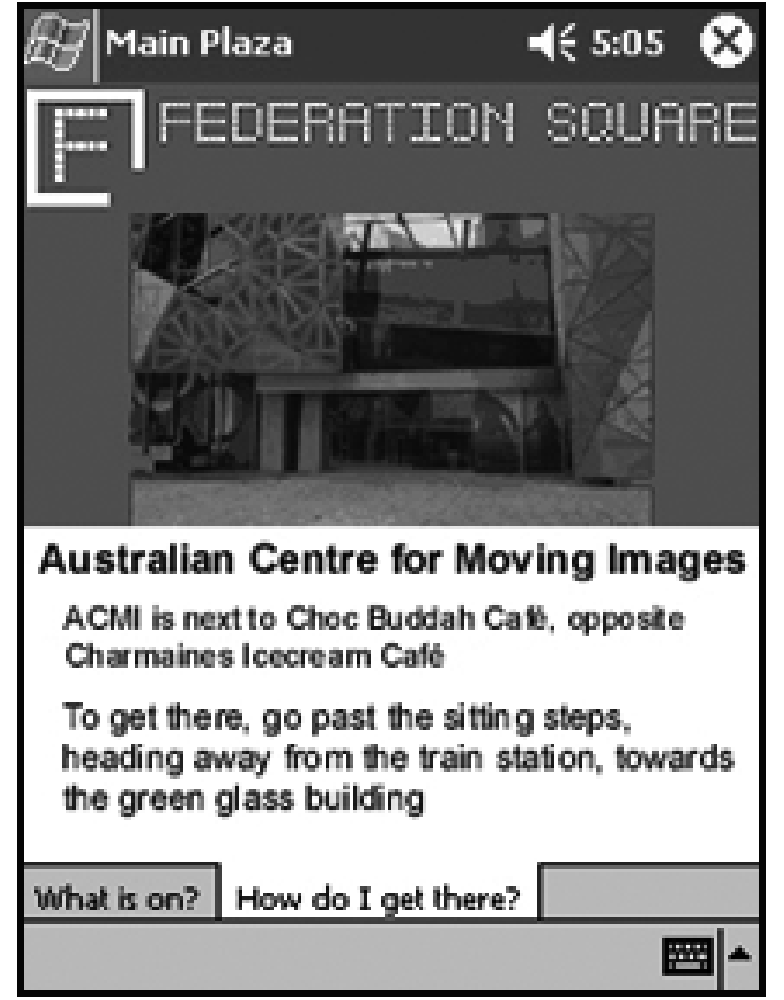

Figure 7. Mobile guide for Federation Square: navigation screen with rich descriptions.

informed by MIRANDA identifying the architectural characteristics of 'activity edges', 'visible surrounds', 'major entrances and paths' and 'no clear paths'. On the navigation screen, the user is presented with rich descriptions derived from the distinctive characteristics of the place. The descriptions used in the guide have two parts. The first describes the location in relation to focal structures. The second part describes the steps taken to get there.

Based on the knowledge from MIRANDA that activities are located on the edges, it follows that we are able to describe selected locations as being 'next to' and/or 'opposite' other locations on the edge of the main plaza. We use these terms in our rich descriptions, thereby indexing locations relative to one another. This is an alternative to absolute location descriptions typically used in mobile guides, for example 'located at the corner of Flinders and Swanston Streets', but holds more meaning to the users of the system, because it makes use of their understanding of the built environment in which they are situated.

The second part of the navigation information describes the path which the user should take to get there. We know from MIRANDA that paths in the main plaza are not clearly marked but that the urban surroundings are visible. 
This allows us to use descriptions, such as 'away from the train station' or 'towards the river', indexing to visible elements of the surrounding city. MIRANDA also tells us that access to the different locations surrounding the main plaza is afforded by major entrances. Based on this, the navigation screen contains an image of the entrance that the user must pass through to get there. Again, this indexes the information given by the mobile guide to the user's physical environment.

Since some of the characteristics of a physical space may sometimes change in accordance to the time of day, season, special events etc., the rich descriptions in the system should be designed so that they can adapt accordingly.

\section{Conclusions}

As digital information becomes a part of the way we operate in the physical world, human-computer interaction with mobile guides can benefit from making use of 'knowledge-in-the-world' by indexing to implicit information cues in the built environment. People have a strong ability to make sense of the physical space in which they are situated and often make use of this when using mobile guides in urban spaces. In order to exploit this ability in mobile guide design, we need to better understand the role of the user's physical environment in defining their context and the contribution of existing information embedded in that environment to people's experience of it. Also, we need to make clear connections between the user's physical surroundings and the information presented on their mobile guide.

This paper has presented the outcome of a field study into the properties of a built environment that contributes to people's experience of their physical context. Synthesizing the outcome of the field study, we have proposed a descriptive framework, MIRANDA, which provides a summarized abstraction of the fundamental architectural and informational features of a built environment. The usefulness of MIRANDA in interface design has been exemplified through the design of an indexical mobile guide system for Federation Square, Melbourne, Australia. Unlike other location-aware guide systems, the proposed design explicitly uses insight into user perceptions of architectural characteristics of the built environment to tailor the information presented to the physical context in which it is being used.

While the outcomes of our analysis are specific to Federation Square, the empirical analysis of an environment used to create the MIRANDA framework could be applied to other cases of mobile guide design for the built environment. In physical spaces where a MIRANDAbased analysis reveals similar architectural characteristics to Federation Square, the specific design ideas of exploring indexicality presented above could also be applied.
The proposed design sketches are currently being implemented as a functional prototype. Through laboratory- and field-based evaluations at Federation Square, the prototype will allow us to validate the usefulness of MIRANDA for informing the design of mobile guides for use in built environments.

In future research, the proposed descriptive framework of MIRANDA should be refined and validated through further studies of other built environments, both similar to, and different from, Federation Square. Additionally, it would be interesting to investigate further the dynamic aspects of physical spaces; characteristics changing according to time of day, season, special events etc. The proposed method does not take this issue explicitly into account during the data collection phase and would have to be extended for this purpose.

\section{Acknowledgements}

The authors thank Steve Howard and Bharat Dave for valuable supervision on the project and comments on the paper. The field study was designed and conducted by the first author, who was also responsible for the data analysis and development of MIRANDA. The design of the mobile guide for Federation Square was done in collaboration with the second author as a part of the 'Design and Use of Context-Aware Mobile Multimedia Systems' and 'Indexical Interaction Design for Context-Aware Mobile Computer Systems' research projects supported by the Danish Technical Research Council (project references 2603-0341 and 26-04-006). Graphical Federation Square illustrations courtesy of Federation Square Management (www.federationsquare.co.au).

\section{References}

Abowd, D., Atkeson, C., Hong, J., Long, S. and Pinkerton, M., 1996, Cyberguide: a mobile context-aware tour guide. Wireless Networks, $\mathbf{3}$, $421-433$.

Agre, P., 2001, Changing places: contexts of awareness in computing. Human-Computer Interaction, 16, 177-192.

Alexander, C., Ishikawa, S., Silverstein, M., Jacobson, M., FiksdahlKing, I. Angel, S., 1977, A Pattern Language: Towns, Buildings, Construction (New York: Oxford University Press).

Andersen, P., 2002, Pervasive computing and space. In Organizational Semiotics: Evolving a Science of Information Systems, L. Kecheng, R.J. Clarke, P.B. Andersen, R. Stamper and El-Sayed Abou-Zeid (Eds.), IFIP TC8/WG8.1, 23-25 July 2002, Montreal, Quebec, Canada (Amsterdam: Kluwer) pp. 133-152.

Aoki, P. and Woodruff, A., 2000, Improving electronic guidebook interfaces using a task-oriented design approach. Proceedings of DIS 2000, New York, USA (New York: ACM), pp. 319-325.

Bertin, J., 1983, Semiology of Graphics (Wisconsin: The University of Wisconsin Press)

Beyer, H. and Holtzblatt, K., 1998, Contextual Design: Defining Consumer-Centered Systems. (San Francisco: Morgan Kaufmann Publishers). 
Bohnenberger, T., Jameson, A., Krüger, A. and Butz, A., 2002, Location-aware shopping assistance: evaluation of a decision-theoretic approach. In Proceedings of Mobile HCI 2002, Pisa, Italy (Berlin: Springer-Verlag), pp. 155-169.

Bornträger, C., Cheverst, K., Davies, N., Dix, A., Friday, A. and SEITZ, J., 2003, Experiments with multi-modal interfaces in a contextaware city guide. Proceedings of Mobile HCI 2003, Udine, Italy (Berlin: Springer-Verlag), pp. 116-130.

Bornträger, C. and Cheverst, K., 2003, Social and technical pitfalls designing a tourist guide system. Proceedings of HCI in Mobile Guides, Udine, Italy, available online at http:/www.mguides.info

Bowman, W.J., 1968, Graphic Communication (New York: John Wiley \& Sons, Inc.).

Bradley, N. and Dunlop, M., 2002, Understanding contextual interactions. Proceedings of Mobile HCI 2002, Pisa, Italy, LNCS (SpringerVerlag), pp. $349-353$.

Borchers, J., 2001, A Pattern Approach to Interaction Design (Chichester: John Wiley \& Sons).

Cheverst, K., Davies, N. and Mitchell, K., 2002, Exploring contextaware information push. Personal and Ubiquitous Computing, 6, 276281.

Cheverst, K., Davies, N., Mitchell, K., Friday, A. and Efstratiou, C., 2000, Developing a context-aware electronic tourist guide: some issues and experiences. Proceedings of CHI'OO, The Hague, Netherlands (New York: ACM), pp. 17-24.

Chincholle, D., Goldstein, M., Nyberg, M. and Erikson, M., 2002, Lost or found? A usability evaluation of a mobile navigation and location-based service. Proceedings of Mobile HCI 2002 Pisa, Italy, LNCS (Springer-Verlag), pp. $211-224$

Crabtree, A., Hemmings, , T. and Rodden, , T. 2001, Domestic legacy and design. Proceedings of the 1st Equator Workshop on Ubiquitous Computing in Domestic Environments, Nottingham, UK (Nottingham: The University of Nottingham), pp. 147-164.

Dieberger, A. and Frank, A., 1998, A city metaphor to support navigation in complex information spaces. Journal of Visual Languages and Computing, 9, $597-622$.

Dix, A., Rodden, T., Davies, N., Trevor, J., Friday, A. and PalfreyMAN, K., 2000, Exploiting space and location as a design framework for interactive mobile systems. ACM Transactions on Computer-Human Interaction, 7, 285-321.

Fithian, R., Iachello, G., Moghazy, J., Pousman, Z. and Stasko, J., 2003, The design and evaluation of a mobile location-aware handheld event planner. Proceedings of Mobile HCI 2003, Udine, Italy (Berlin: Springer-Verlag), pp. $145-160$.

Gamma, E., 1997, Going beyond objects with design patterns. Proceedings ECOOP '97 - Object-Oriented Programming: 11th European Conference, June 1997, Jyväskylä, Finland, p. 530.

Graham, C. and Kueldskov, J., 2003, Indexical representations for context-aware mobile devices. Proceeding of The IADIS e-Society Conference, Lisbon, Portugal, pp. 373-380.

HAKkila, J. and Hexel, R., 2003, Interaction in location-aware messaging in a city environment. Proceedings of OZCHI2003: New Directions in Interaction: Information Environments, Media \& Technology, December 2003, Brisbane, Australia (Brisbane: CHISIG), pp. 84-93.

HARrison, S. and Dourish, P., 1996, Re-placing space: the roles of place and space in collaborative systems. Proceedings of the ACM CSCW 96 Conference on Computer-Supported Cooperative Work (New York: ACM), pp. $67-76$.

Hermann, F. and Heidmann, F., 2002, User requirement analysis and interface conception for a mobile, location-based fair guide. Proceedings of Mobile HCI 2002, Pisa, Italy, LNCS (Springer-Verlag), pp. 388-392.
Holland, S. and Morse, D.R., 2001, AudioGPS: spatial audio in a minimal attention interface. Proceedings of Mobile HCI 2001: Third International Workshop on Human Computer with Mobile Devices, Lille, France, available online at http://www.mobilechi.org

Iacucci, G., Kela, J. and Pehonen, P., 2004, Computational support to record and re-experience visits. Personal and Ubiquitous Computing, 8, $100-109$.

Ingram, R., Benford, S. and Bowers, J., 1996, Building virtual cities: applying urban planning principles to the design of virtual environments. Proceedings of ACM symposium on Virtual Reality Software and Technology (VRST96), July, 1996 (ACM), pp. 83-91.

KJeldskov, J., 2002, Just-in-place: information for mobile device interfaces. Proceedings of Mobile HCI 2002, Pisa, Italy (Berlin: SpringerVerlag), pp. 271-275.

Kueldskov, J., Howard, S., Murphy, J., Carroll, J., Vetere, F. and Graham, C., 2003, Designing TramMate - a context aware mobile system supporting use of public transportation. Proceedings of $D U X$ 2003: Designing for user experiences, San Francisco, CA, USA (ACM).

Kolari, J. and Virtanen, T., 2003, In the zone: views through a contextaware mobile portal. Proceedings of HCI in Mobile Guides, Udine, Italy.

Kuluu, E. and KAAsinen, E., 2002, Route guidance using a 3D city model on a mobile device. Proceedings of Mobile Tourism Support, Pisa, Italy, available online at http://www.mguides.info

Laakso, K., Guesdal, O. and Sulebak, J.R., 2003, Tourist information and navigation support by using $\mathrm{D}$ maps displayed on mobile devices. Proceedings of HCI in Mobile Guides, Udine, Italy, available online at http://www.mguides.info

LyNCH, K., 1960, The Image of the City (Cambridge, Mass.: MIT Press).

McCullough, M., 2001, On typologies of situated interaction. HumanComputer Interaction, 16, $337-$

Millen, D.R., 2000, Rapid ethnography: time deepening strategies for HCI field research. Proceedings of DIS 2000, Brooklyn, New York, USA (New York: ACM), pp. 280-286.

Nakanishi, H., Koizumi, S., Ishida, T. and Ito, H., 2004, Transcendent communication: location-based guidance for large-scale public spaces. Proceedings of CHI 2004, Vienna, Austria (ACM), pp. 655-662.

Norman, D., 1990, The Psychology of Everyday Things (New York: Basic Books).

Opperman, R. and Specht, M., 2000, A context-sensitive nomadic information system as an exhibition guide. Proceedings of Handheld and Ubiquitous Computing Second International Symposium, Bristol, UK (Berlin: Springer-Verlag), pp. 127-142.

PAAY, J., 2003, Understanding and modeling physical environments for mobile location aware information services. Proceedings of Mobile $\mathrm{HCI}$ 2003, Udine, Italy (Berlin: LNCS, Springer-Verlag), pp. 405-410.

Paulos, E. and Goodman, E., 2004, The familiar stranger: anxiety, comfort and play in public places. Proceedings of CHI 2004, Vienna, Austria (New York: ACM), pp. 223-230.

Persson, P., Espinoza, F., SAndin, A. and Coster, R., 2002, GeoNotes: a location-based information system for public spaces. Proceedings of Mobile HCI 2002, Pisa, Italy, LNCS (Berlin: LNCS, Springer-Verlag), pp. $151-173$.

Pospischil, G., Umlauft, M. and Michlmayr, E., 2002, Designing LoL@, a mobile tourist guide for UMTS. Proceedings of Mobile HCI 2002, Pisa, Italy (Springer-Verlag), pp. 140-154.

Randell, C. and Muller, H., 2000, The shopping jacket: wearable computing for the consumer. Personal and Ubiquitous Computing, 4, $241-244$. 
Ricci, F., Cavada, D. and Nguyen, Q.N., 2002, Integrating travel planning and on-tour support in a case-based recommender system. Proceedings of Mobile Tourism Support, Pisa, Italy, available online at http://www.mguides.info

Rocchi, C., Stock, O. and Zancanaro, M., 2003, Semantic-based multimedia representations for the museum experience. Proceedings of HCI in Mobile Guides, Udine, Italy, available online at http:// www.mguides.info

Sparacino, F., Davenport, G. and Pentland, A., 2000, A city of news: cataloguing the world wide web through virtual architecture. KOS, Aug-Sept 2000, pp. 179-180.

Schmidt-Belz, B. and Poslad, S., 2003, User validation of a mobile tourism service. Proceedings of HCI in Mobile Guides, Udine, Italy, available online at http://www.mguides.info

Tamminen, S., Oulasvirta, A., Toiskallio, K. and Kankainen, A., 2003, Understanding mobile contexts. Proceedings of Mobile HCI 2003, Udine, Italy (Berlin: LNCS, Springer-Verlag), pp. 17-31.
Tidwell, J., 1999, Common Ground: A Pattern Language for HumanComputer Interface Design. Available online at: http://www.mit.edu/ $\sim$ jtidwell/interaction_patterns.html

Tufte, E., 1990, Envisioning Information (Cheshire, Conn.: Graphics Press).

Vainio, T., Kotala, O., Rakkolainen, I. and Kupila, H., 2002, Towards scalable user interfaces in D city information systems. Proceedings of Mobile HCI 2002, Pisa, Italy (Berlin: LNCS, Springer-Verlag), pp. $354-$ 358.

VInSON, N.G., 1999, Design guidelines for landmarks to support navigation in virtual environments. Proceedings of CHI 1999, Pittsburgh, PA, USA (New York: ACM), pp. 278-285. 